\title{
Self-consistent integral equation theory for solutions of finite extensible semiflexible polyelectrolyte chains
}

\author{
T. Hofmann \\ Abteilung Theoretische Physik, Universität Ulm, D-89069 Ulm, Germany \\ R. G. Winkler ${ }^{\mathrm{a})}$ \\ Institut für Festkörperforschung, Forschungszentrum Jülich, D-52425 Jülich, Germany \\ P. Reineker \\ Abteilung Theoretische Physik, Universität Ulm, D-89069 Ulm, Germany
}

(Received 28 October 2002; accepted 13 January 2003)

\begin{abstract}
We investigate the structural and conformational properties of solutions containing semiflexible polyelectrolyte chains using a self-consistent integral equation theory approach. A one-component system is considered where the polyelectrolyte chains interact with each other via a Debye-Hückel potential. Nonelectrostatic interactions among the polymers are taken into account by a self-consistently determined solvation potential. The conformational properties of the polymer chain are determined from a variational calculation with a semiflexible reference chain. The finite chain extensibility is taken into account by constraints for the bond lengths and bond angles using Lagrangian multipliers. The scaling relation for the size of an isolated semiflexible chain with respect to chain length exhibits a transition from rodlike to excluded volume type for a given Debye screening length. For flexible chains in solution, the theory provides conformational properties which are in excellent agreement with computer simulation results. The bare chain stiffness has a pronounced influence on the conformational and structural properties of the solution. In the semidilute regime a pronounced liquidlike order is obtained for flexible polyelectrolyte chains which diminishes with increasing bare persistence length. This process is accompanied by a shift of the structural peaks to smaller length scales. (C) 2003 American Institute of Physics.
\end{abstract}

[DOI: $10.1063 / 1.1557472]$

\section{INTRODUCTION}

Polyelectrolytes are polymers consisting of monomers with ionizable groups. These ionizable groups dissociate when the polyelectrolytes are dissolved in a polar solvent such as water. The remaining charged particles, i.e., large polyions and small counterions, interact via long-range Coulomb interactions. This leads to a wide variety of effects which are not present in solutions of uncharged polymers. Polyelectrolytes play a fundamental role in our everyday life. For example, two of the most well known polymers, DNA and RNA, are polyelectrolytes. Furthermore, many other biopolymers like proteins are polyelectrolytes. In addition, a large class of synthetic polyelectrolytes exists as well, which are utilized in a wide range of technical applications such as water purification, stabilization of gels, or superabsorbers. ${ }^{1-5}$

Despite significant experimental and theoretical efforts over the last decades, ${ }^{1,2,5-7}$ many properties of polyelectrolytes are, in comparison to neutral polymers, still poorly understood. ${ }^{5,8-12}$ The coupling of various length scales introduced by the long-range nature of the Coulomb interaction, counterion condensation, and screening effects render polyelectrolytes difficult to study both from the theoretical and experimental point of view. In particular the coupling of the different length scales poses major problems for the applica-

${ }^{a)}$ Electronic mail: r.winkler@fz-juelich.de tion of renormalization group theories and scaling ideas, which proved to be very successful for neutral polymers. ${ }^{13-15}$ Computer simulations provide valuable insight into the structural properties of polyelectrolyte solutions but require special techniques to treat the long-range Coulomb interaction, e.g., Ewald summation, ${ }^{16}$ and hence are often limited to small systems with short chains and/or low densities. ${ }^{17-25}$ Scattering experiments, on the other hand, suffer from trace impurities and very low scattering intensities encountered in dilute solutions. Therefore, experimental data for the properties of single chains are lacking. ${ }^{5,26-35}$ Moreover, often controversial results are reported when using different experimental methods. ${ }^{5}$

Liquid state theory based upon the polymer-referenceinteraction-site model (PRISM) ${ }^{36,37}$ offers another theoretical approach to polyelectrolyte solutions. PRISM theory is an extension of the Ornstein-Zernike equation ${ }^{38}$ of atomic systems to molecular systems by taking the connectivity of the sites of a molecule into account by the chain structure factor. The chain conformations and all intramolecular interactions appear in this theory solely via the chain structure factor. During the last years, PRISM has successfully been used to characterize the properties of systems composed of rodlike polyelectrolytes. ${ }^{39-42}$ Particularly Shew and Yethiraj contributed to the understanding of polyelectrolyte solutions and demonstrated that the results of the PRISM approach are usually in good agreement with results from computer simu- 
lations or experiments. ${ }^{42-46}$ Polyelectrolytes cover the whole range of stiffnesses from flexible to rodlike polymers depending upon the chain length or the solution properties like the salt concentration. As a consequence, the structure of a solution and the conformations of the polyelectrolyte chains are strongly coupled. To account for this coupling, the solutions of the PRISM equations and the corresponding conformations of the polyelectrolytes have to be determined simultaneously. This is achieved by a recently developed extension of the PRISM theory. ${ }^{43,47-56}$ Here, the nonpairwise intermolecular many chain interactions of a particular chain are cast into an effective pairwise intramolecular solvation potential which is determined self-consistently. This so called medium induced potential can be expressed by correlation functions calculated with the PRISM theory. ${ }^{57-60}$

In this paper we apply the self-consistent PRISM theory to semiflexible polyelectrolyte solutions taking into account the finite chain extensibility by constraints for the bond angles and bond lengths. At infinite dilution, our results for the dependence of the mean square end-to-end distance on the Debye screening length of fully flexible chains are in excellent agreement with computer simulations. Considering the scaling behavior of the mean square end-to-end distance with chain length at a fixed Debye screening length, we find a crossover from a rodlike to self-avoiding-walk behavior at low salt concentrations. ${ }^{61}$ Including the medium-induced potential, we again find excellent agreement between computer simulation results and our calculations for the density dependence of the chain conformations. In this context, we conclude that the observed contraction of the chains with increasing density is dominated by the screening of the Coulomb interactions and packing effects are almost negligible. At dilute solutions, an increase of the Bjerrum length leads to a rapid increase of the chain size up to rodlike conformations. In contrast, at high densities the chain size is independent of the Bjerrum length due to screening effects. For semiflexible chains we find results which contradict the findings of Ref. 54. Our calculations reveal that the chain conformations not only depend on the chain stiffness in dilute but also in semidilute solutions. In particular, the chains contract to the size of the corresponding uncharged semiflexible chain for high densities. Chain stiffness is also reflected in the structural properties of a solution which we discuss using the pair correlation function.

The rest of the paper is organized as follows: In Sec. II we present the chain model and outline the variational procedure to calculate the chain conformations. In Sec. III we describe the self-consistent PRISM theory. Results are presented in Sec. IV for single chains and in Sec. V for many chain systems. Finally, in Sec. VI we summarize our results.

\section{POLYELECTROLYTE CONFORMATIONS}

The self-consistent solution of the PRISM equations requires knowledge of the conformations of a polyelectrolyte chain. We will use an analytical approach to derive the necessary expressions. Alternatively, simulation results can be exploited to obtain the required input. ${ }^{43}$ In this section, we will outline the applied chain model.

\section{A. Chain model}

The PRISM equations use a site representation of a polymer chain. To be consistent, we will use a discrete semiflexible chain model. ${ }^{62}$

The chain is considered as a one dimensional arrangement of $N+1$ mass points with equal masses $m$. The positions of the points are denoted by $\mathbf{r}_{i}, i=0, \ldots, N$. To remove the translational degrees of freedom, the point $\mathbf{r}_{0}$ is fixed at the origin of the reference frame. The other mass points are subject to the constraints

$$
\begin{aligned}
& \sum_{i=2}^{N-1}\left\langle\mathbf{R}_{i}^{2}\right\rangle=(N-2) l^{2}, \\
& \left\langle\mathbf{R}_{1}^{2}\right\rangle=\left\langle\mathbf{R}_{N}^{2}\right\rangle=l^{2}, \\
& \sum_{i=1}^{N-1}\left\langle\left(\mathbf{R}_{i}-\mathbf{R}_{i+1}\right)^{2}\right\rangle=2(N-1) l^{2}(1-t) .
\end{aligned}
$$

Here the $\mathbf{R}_{i}=\mathbf{r}_{i}-\mathbf{r}_{i-1}$ are bond vectors and $l$ is the bond length. Equations (1) and (2) capture the connectivity of the mass points along the chain contour and guarantee the finite chain extensibility. The chain ends have to be taken into account explicitly to obtain correct results in the rod limit. ${ }^{62}$ Equation (3) describes bond angle restrictions which result in an intrinsic chain stiffness. The parameter $t$ is proportional to the average cosine of the angle between successive bonds, whereby $t=0$ corresponds to a totally flexible chain (Gaussian chain) and $t=1$ describes a rigid rod. Using the maximum entropy principle, we find the configurational partition function $Z=\int \exp \left(-\beta H_{0}\right) d^{3 N} x$ with the Hamiltonian

$$
\beta H_{0}=\nu \sum_{i=2}^{N-1} \mathbf{R}_{i}^{2}+\nu_{0}\left(\mathbf{R}_{1}^{2}+\mathbf{R}_{N}^{2}\right)+\frac{\epsilon}{2} \sum_{i=1}^{N-1}\left(\mathbf{R}_{i}-\mathbf{R}_{i+1}\right)^{2} .
$$

The Lagrangian multipliers $\nu, \nu_{0}$, and $\epsilon$ take into account the constraints (1)-(3) and are given by ${ }^{62}$

$$
\nu=\frac{3}{2 l^{2}} \frac{1-t}{1+t}, \quad \nu_{0}=\frac{3}{2 l^{2}} \frac{1}{1+t}, \quad \epsilon=\frac{3}{l^{2}} \frac{t}{1-t^{2}} .
$$

In the continuum limit, the model is identical to a path integral representation of a semiflexible chain as proposed by various authors. ${ }^{62-66}$ The above values of the Lagrangian multipliers are correct only as long as there are no other interactions present. Additional interactions, like external electric or flow fields or forces acting at the chain ends to stretch a chain, require the adjustment of the Lagrangian multipliers to fulfill the constraints (1)-(3). ${ }^{67-70}$

Since the Hamiltonian $H_{0}$ is quadratic in the bond vectors various averages can be obtained analytically. In particular, the mean square end-to-end distance is given by

$$
\left\langle\mathbf{R}_{E}^{2}\right\rangle=\left\langle\left(\mathbf{r}_{N}-\mathbf{r}_{0}\right)^{2}\right\rangle=N l^{2}\left(\frac{1+t}{1-t}+\frac{2 t}{N} \frac{t^{N}-1}{(t-1)^{2}}\right)
$$

and the structure factor reads as 


$$
\begin{aligned}
\omega(k)= & \frac{1}{N+1} \sum_{i, j=0}^{N} \exp \left[-\frac{k^{2}|j-i| l^{2}}{6}\left(\frac{1+t}{1-t}\right.\right. \\
& \left.\left.+\frac{2 t}{|j-i|} \frac{t^{|j-i|}-1}{(t-1)^{2}}\right)\right] .
\end{aligned}
$$

To describe a polyelectrolyte, we assign a charge $Z e$ to each of the $N+1$ monomers. Since we account for the counterions and added salt only within the Debye-Hückel approximation, the Hamiltonian $H_{0}$ has to be extended by the potential

$$
\beta V_{C}=Z^{2} l_{\mathrm{B}} \sum_{i=0}^{N} \sum_{j=i+1}^{N} \frac{e^{-\kappa\left|\mathbf{r}_{i}-\mathbf{r}_{j}\right|}}{\left|\mathbf{r}_{i}-\mathbf{r}_{j}\right|},
$$

where $l_{\mathrm{B}}=\beta e^{2} / \varepsilon$ is the Bjerrum length, $\kappa=\sqrt{4 \pi l_{\mathrm{B}} \rho}$ is the inverse Debye screening length, $\rho$ is the monomer density or, for systems with added salt, the density of the monomers and the saltions, and $\beta=1 / k_{\mathrm{B}} T$ with $T$ the temperature and $k_{\mathrm{B}}$ the Boltzmann factor. The solvent is considered as a continuous background with the dielectric constant $\varepsilon$.

In addition, other potentials, like the excluded volume potential, can be taken into account. The total Hamiltonian of our system is then given by $H=H_{0}+V$, where $V$ includes all potential energies.

The presence of the potential $V$ requires an adjustment of the Lagrangian multipliers. Unfortunately, the partition function with the Hamiltonian $H$ cannot be calculated in closed form in general. Thus, we have to resort to an approximate calculation. The details of the calculations will be presented in Secs. II B and II C.

A similar description of a semiflexible polyelectrolyte chain based on a continuum model has been used in Ref. 61. The Debye-Hückel potential exhibits a singularity in this limit, since the distance between two charges along the chain contour becomes zero. To overcome this problem a perturbation expansion is used to remove the singularity ${ }^{61}$ or a cut-off is introduce ${ }^{71}$ which determines a smallest possible length scale. Such approximations are not necessary for the discrete model used in this article.

\section{B. Perturbation calculation}

To calculate the conformational properties of the semiflexible polyelectrolyte chain, we resort to the approximation scheme proposed by Edwards and Singh. ${ }^{72}$ Alternatively, the Gibbs-Bogoliubov variational method could be exploited. ${ }^{38,71,73}$ Our calculations show, however, that the results obtained by the Edwards-Singh approach are in better agreement with computer simulations.

The Edwards-Singh approach is a perturbation calculation with respect to a reference chain. The basic idea is to determine free parameters of an analytically tractable reference chain in such a way that its conformational properties agree with those of the original chain. We use the semiflexible chain with the Hamiltonian (4), where the stiffness $t$ is replaced by the reference value $t_{R}$, as a reference system. To adjust the conformations of the two chains, we require that their mean square end-to-end distances are equal, i.e.,

$$
\left\langle\mathbf{R}_{E}^{2}\right\rangle=\left\langle\mathbf{R}_{E}^{2}\right\rangle_{R}
$$

Here $\langle\cdots\rangle_{R}$ denotes averages calculated with the reference Hamiltonian $H_{R}$, whereas $\langle\cdots\rangle$ means that the average is performed with the full Hamiltonian $H$. Expanding $\left\langle\mathbf{R}_{E}^{2}\right\rangle$ in terms of $\left\langle\mathbf{R}_{E}^{2}\right\rangle_{R}$ and $\left\langle H-H_{R}\right\rangle_{R}$ up to first order in the perturbation $\left(H-H_{R}\right)$ leads to the following equation:

$$
\left\langle\mathbf{R}_{E}^{2}\right\rangle_{R}\left\langle H-H_{R}\right\rangle_{R}-\left\langle\mathbf{R}_{E}^{2}\left(H-H_{R}\right)\right\rangle_{R}=0
$$

for the stiffness $t_{R}$.

Since the reference Hamiltonian is quadratic, the averages of Eq. (10) can be calculated analytically. The results are presented in Appendix A. The structure factor, required for the PRISM calculations, follows from Eq. (7) via replacing $t$ by $t_{R}$.

\section{Lagrangian multiplier}

The equation to determine the stiffness $t_{R}$ (A3) includes the Lagrangian multipliers $\nu, \nu_{0}$, and $\epsilon$ of the original chain. As pointed out in the last section, these parameters have to be determined such that the constraints (1)-(3) are satisfied. Since no closed analytical solution for the partition function can be obtained, approximation schemes are necessary to fix the multipliers. We tried several approximation schemes such as a perturbation expansion or adjustment to a stretched reference chain. The first approach fails usually, because the changes in the chain conformations are generally not small as required for a perturbation.

The semiflexible chain is a coarse-grained model of a polymer chain and the Lagrangian multiplier $\epsilon$ determines the stiffness of the chain. Without potential energy, $\epsilon$ is a measure for the bare persistence length. Intermolecular interactions may change the total persistence length but not the bare persistence length, which is an intrinsic property of a polymer chain. Therefore, we consider $\epsilon$ to be independent from the potential $V$. The Lagrangian multipliers $\nu$ and $\nu_{0}$ keep the bond lengths at their average values. (To strictly fulfill this condition would actually require $N$ Lagrangian multipliers - one for every bond. Only for the Hamiltonian $H_{0}$ all the multipliers, except those at the chain ends, are equal. In general, all multipliers are different. ${ }^{68-70}$ ) Since variations of the potential energy $V$ lead to changes in the bond forces, $\nu$ and $\nu_{0}$ have to be adjusted to prevent variations in the chain contour length $L=N l$ and hence overstretching of the chain.

To obtain an estimate of $\nu$ and $\nu_{0}$, we use a stretched semiflexible chain as a reference chain. One way to describe the deformation of a polymer chain is to introduce the constraint

$$
\left\langle\mathbf{r}_{N}^{2}\right\rangle=\mathbf{a}^{2},
$$

for the chain end point $\left(\mathbf{r}_{N}\right)$. The partition function with this additional constraint can be calculated. The result is presented in Appendix B. Considering the Lagrangian multipliers $\widetilde{\nu}$ and $\widetilde{\nu}_{0}$ of the deformed chain, we see that they depend on the extension $|\mathbf{a}|$. The calculation of the desired multipliers proceeds then as follows: Using the stiffness $t_{R}$ obtained from the Edwards-Singh approach, the mean square end-toend distance $\left\langle\mathbf{R}_{E}^{2}\right\rangle_{R}$ is calculated and set equal to $\mathbf{a}^{2}$. The Lagrangian multipliers $\widetilde{\nu}$ and $\widetilde{\nu}_{0}$ are then calculated by solv- 
ing Eqs. (B8), (B9). Finally, we set $\nu=\widetilde{\nu}$ and $\nu_{0}=\widetilde{\nu}_{0}$. Equation (10) is then solved again to obtain an improved value for $t_{R}$. With this value new multipliers are determined. The procedure is repeated until convergence has been achieved.

\section{SELF-CONSISTENT PRISM THEORY}

The polymer reference interaction site model (PRISM) is an integral equation theory for molecular systems. It follows from the Ornstein-Zernike equation ${ }^{38}$ for atomic systems by taking into account the connectivity of the sites of a linear molecule. This is achieved by incorporating the intramolecular structure factor $\omega(k)$ in the appropriate equations. The PRISM equations then relate the total correlation function $h(r)$, the direct correlation function $c(r)$, and the intramolecular correlation functions, represented by $\omega(k)$, with each other. Structural properties of a solution, like the pair correlation function $g(r)$ or the static structure factor $S(k)$, can be obtained easily from the total correlation function $h(r)$ via $g(r)=h(r)+1$ and $S(k)=\omega(k)+\rho h(k)$, respectively. If all monomers of the chain are considered to be equivalent and hence chain end effects are neglected, the structural properties of the solutions can be described by a single correlation function in contrast to $N^{2}$ different correlation functions when all $N$ monomers of the chain are treated individually. In Fourier space, the PRISM equation is given by

$$
h(k)=\omega(k) c(k) \omega(k)+\rho \omega(k) c(k) h(k),
$$

where $\rho$ is the monomer density. To solve the PRISM equation a relation is required which connects the total and direct correlation function. Various such closure relations have been proposed. ${ }^{37,58,74-76}$ In this work we use the Laria-WuChandler (LWC) closure which proved to be adequate for threadlike polyelectrolytes and is given by

$$
\begin{aligned}
\omega(r) * c(r) * \omega(r)= & -\omega(r) * \beta v(r) * \omega(r)+h(r) \\
& -\ln g(r),
\end{aligned}
$$

where the asterisks denote convolution integrals and $v$ is the intermolecular interaction potential.

In this theory, the chain conformations solely enter via the intramolecular structure factor $\omega(k)$. Thus, no detailed knowledge about intramolecular potentials is necessary.

To calculate the intramolecular structure factor, the intermolecular many chain interactions are approximated by a solvation potential. This medium-induced potential $(W)$ is assumed to be pairwise additive and has to be determined in a self-consistent manner. ${ }^{43,47-56}$ An approximate expression in terms of the direct correlation function and the static structure factor $S=\omega(k)+\rho h(k)$ reads $^{57-60,77}$ as

$$
\beta W_{i, j}(k)=-\rho c(k) S(k) c(k) .
$$

The total intramolecular potential $V$ is then given by

$$
V(\mathbf{r})=\sum_{i=0}^{N} \sum_{j=i+1}^{N}\left[Z^{2} l_{B} \frac{e^{-\kappa\left|\mathbf{r}_{i}-\mathbf{r}_{j}\right|}}{\left|\mathbf{r}_{i}-\mathbf{r}_{j}\right|}+\beta W_{i, j}\left(\left|\mathbf{r}_{i}-\mathbf{r}_{j}\right|\right)\right] .
$$

In the following, we will consider systems with monovalent charges, i.e., $Z=1$.

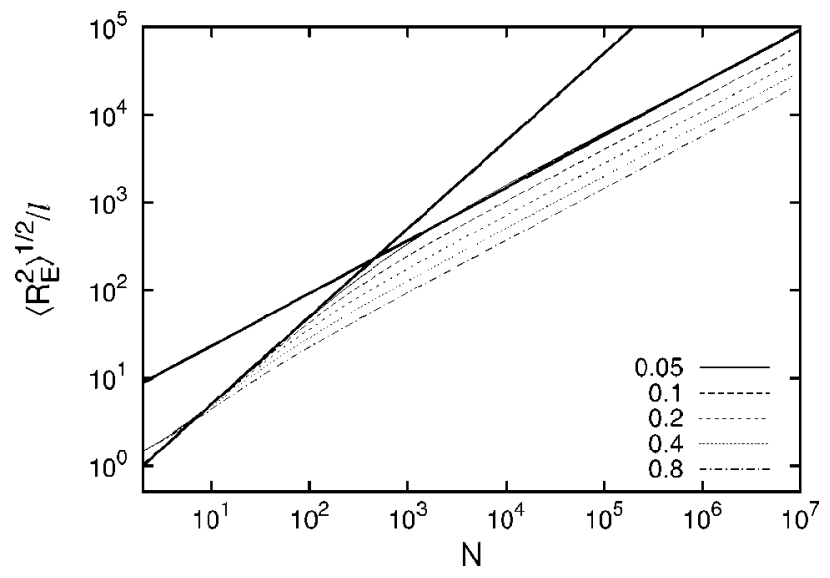

FIG. 1. Root mean square end-to-end distance of flexible polyelectrolyte chains as a function of the chain length for $l_{\mathrm{B}}=0.5 l$. The inverse Debye screening length $\kappa$ varies from 0.05 to 0.8 (top to bottom). The slopes of the two straight lines are 1 and $3 / 5$, respectively.

\section{SINGLE CHAIN RESULTS}

A single chain system corresponds to the limit $\rho \rightarrow 0$ and the medium-induced potential is zero. The potential energy $V$ is then equal to the Debye-Hückel potential and the solution of the PRISM equation is not necessary to calculated the conformational properties. Hence, our system corresponds to the study of Ref. 61 aside from the fact that we consider a discrete model and a finite extensible polyelectrolyte chain.

Considering the scaling behavior of the mean square end-to-end distance of a flexible chain $(t=0)$ with chain length, we find various regimes depending on the Debye screening length. Figure 1 displays $\left\langle\mathbf{R}_{E}^{2}\right\rangle$ as a function of chain length and inverse Debye screening length for $l_{\mathrm{B}}$ $=0.5 l$. For large $\kappa$, i.e., small screening lengths, $\left\langle\mathbf{R}_{E}^{2}\right\rangle$ exhibits self-avoiding-walk behavior $\left(\left\langle\mathbf{R}_{E}^{2}\right\rangle \sim N^{1.2}\right)$ for almost all chain lengths. This is a consequence of the fact that the Debye-Hückel potential is effectively a short range potential when the chain length exceeds the Debye screening length. A decrease of $\kappa$ leads to an increase of the screening length. For a certain range of chain lengths, a second scaling regime appears with $\mathbf{R}_{E}^{2} \sim N^{2}$. (The exponent slightly depends on $\kappa$, but the limiting value is 2.) For large chain lengths again self-avoiding-walk behavior is assumed. Although in the intermediate regime the scaling relation is close to the scaling behavior of a rod, the value of $\left|\mathbf{R}_{E}\right|$ is significantly smaller than for a rodlike conformation. These results are in agreement with the findings presented in Refs. 61 and 78.

Recently, computer simulation results for the mean square end-to-end distance as a function of $\kappa$ have been published for a broad range of chain lengths. ${ }^{79}$ Figure 2 compares results of our analytical approach (lines) with the simulation data (symbols) for the Bjerrum length $l_{\mathrm{B}}=l$. As is obvious from the figure, the theoretical approach captures the transition from the extended state of the polymer (almost rodlike) to a collapsed state (Gaussian coil) with increasing $\kappa$. Considering the quantitative agreement between the two sets of data, the overall agreement is very good, even for longer chains. (It should be kept in mind that there is no free parameter in the theory.) However, for long chains we find 


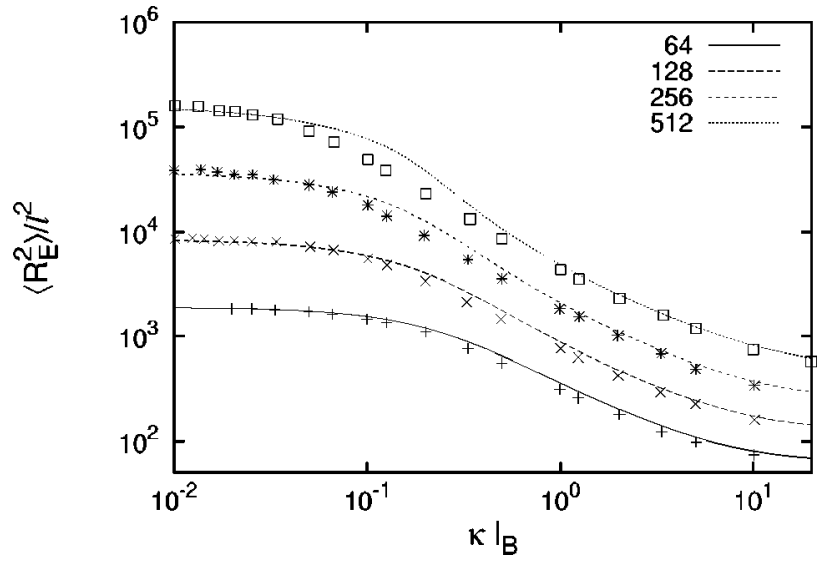

FIG. 2. A comparison between results of this work (lines) and computer simulations (dots) (Ref. 79) for the mean square end-to-end distance as a function of the inverse screening length $\kappa$ for fully flexible chains. The chain length varies from $N=64$ to $N=512$ (bottom to top) at constant Bjerrum length $l_{\mathrm{B}}=1.0 l$

discrepancies between the two approaches for $\kappa$ values in the transition regime from an extended to a collapsed chain. At large and small $\kappa$, the agreement is still excellent.

The increase of the chain length at a constant Bjerrum length leads to an increase of the Coulomb interaction. Hence, the observed discrepancies seem to point to a failure of our approach at large interaction strengths. Whether this is related to the applied approximations or points to a failure of the semiflexible chain description of the polyelectrolyte chain remains to be investigated.

\section{RESULTS FOR MANY CHAIN SYSTEMS}

In this section we will present results taking into account the medium-induced potential. At first we will study the conformational properties of the polyelectrolyte chains and then discuss the structure of the solution.

\section{A. Fully flexible chain}

Extensive computer simulations for flexible chains ( $t$ $=0$ ) in solutions are available..$^{20,43}$ The present theory is in quantitative agreement with the simulation results. Figure 3 displays the root mean square end-to-end distance of a fully flexible chain as a function of density and for various chain lengths. Our approach is in better agreement with the simulation data than the results of the theories of Yethiraj presented in Refs. 52 and 53. At low concentrations, the chains are extended and they contract with increasing density. For very high densities, the dimension of an uncharged chain is assumed. The medium-induced potential leads to smaller end-to-end distances than obtained without such a potential, particularly for high concentrations. As is obvious from the figure, the critical density, where the chains start to contract, moves to smaller densities with increasing chain length. The decrease of the mean square end-to-end distance with increasing density may be explained by two different effects. An increase of the monomer density implies a decrease of the Debye screening length. Therefore, the intramolecular Coulomb interaction is strongly screened at high densities, leading to more compact conformations. On the other hand,

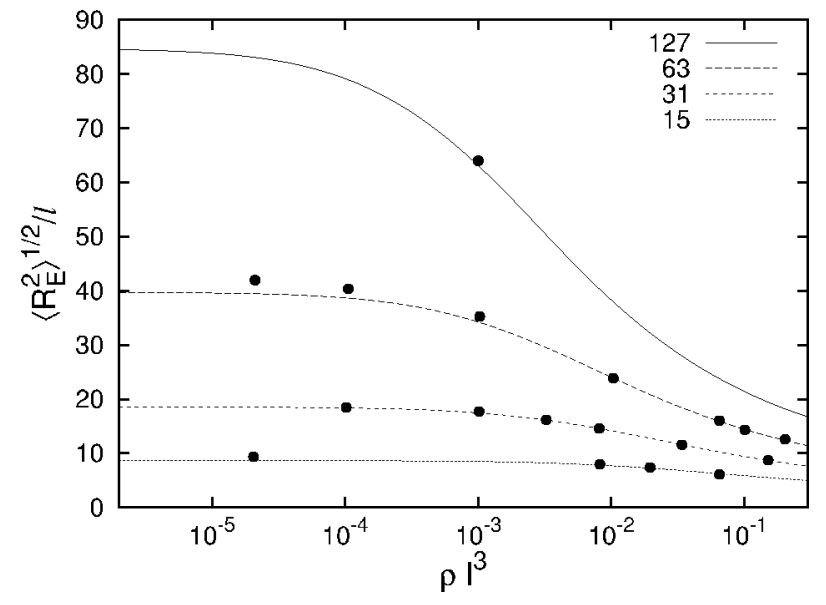

FIG. 3. A comparison between the density dependencies of the root mean square end-to-end distance of fully flexible chains obtained from this work (lines) and from computer simulations (symbols) (Refs. 43, 81). The chain length varies from $N=15$ to $N=127$ and the Bjerrum length is fixed to $l_{\mathrm{B}}$ $=0.833 l$.

more compact structures may form when the overlap density is reached or exceeded, respectively. The question arises as to which of the two effects is the dominating effect for chain compression.

If the chain contraction is governed by the packing effect, the chains should start to contract for densities larger than the overlap density $\rho^{*}$, defined by

$$
\rho^{*}=\frac{N}{\left\langle\mathbf{R}_{g}^{2}\right\rangle^{3 / 2}} .
$$

Here, we use the radius of gyration $\mathbf{R}_{g}$ to characterize the average volume occupied by a chain, where $\left\langle\mathbf{R}_{g}^{2}\right\rangle$ is a function of density. Figure 4 (top) depicts the dependence of the mean square end-to-end distance on the scaled density $\rho / \rho^{*}$ for the same set of parameters as in Fig. 3. The chains start to contract for densities much smaller than the overlap density $\rho^{*}$. Therefore packing effects seem to play a minor role for chain contraction. On the other hand, if the screening effect is dominant, the chains should start to contract when the screening length, which depends directly on the density, is of the order of a typical chain dimension, e.g., the radius of gyration. This allows us to define a critical screening density via

$$
\kappa=\sqrt{4 \pi l_{\mathrm{B}} \rho_{c}}=\left\langle\mathbf{R}_{g}^{2}\right\rangle^{-1 / 2} .
$$

As is obvious from Fig. 4 (bottom), chains of various lengths start to contract when the density is close to the critical screening density. Therefore, the screening of the Coulomb potential with increasing density is the dominant effect for the decrease of the chain size. This is confirmed by calculations of the mean square end-to-end distance for a single chain, i.e., in the limit $\rho \rightarrow 0$, where packing effects are irrelevant. Similar results are obtained for both systems. Only at high densities, packing effects play an important role.

Since overstretching of the chains is prevented in our model by adjusting the Lagrangian multipliers, it is possible to discuss the density dependence of the mean square endto-end distance for larger Bjerrum lengths than the ones used 

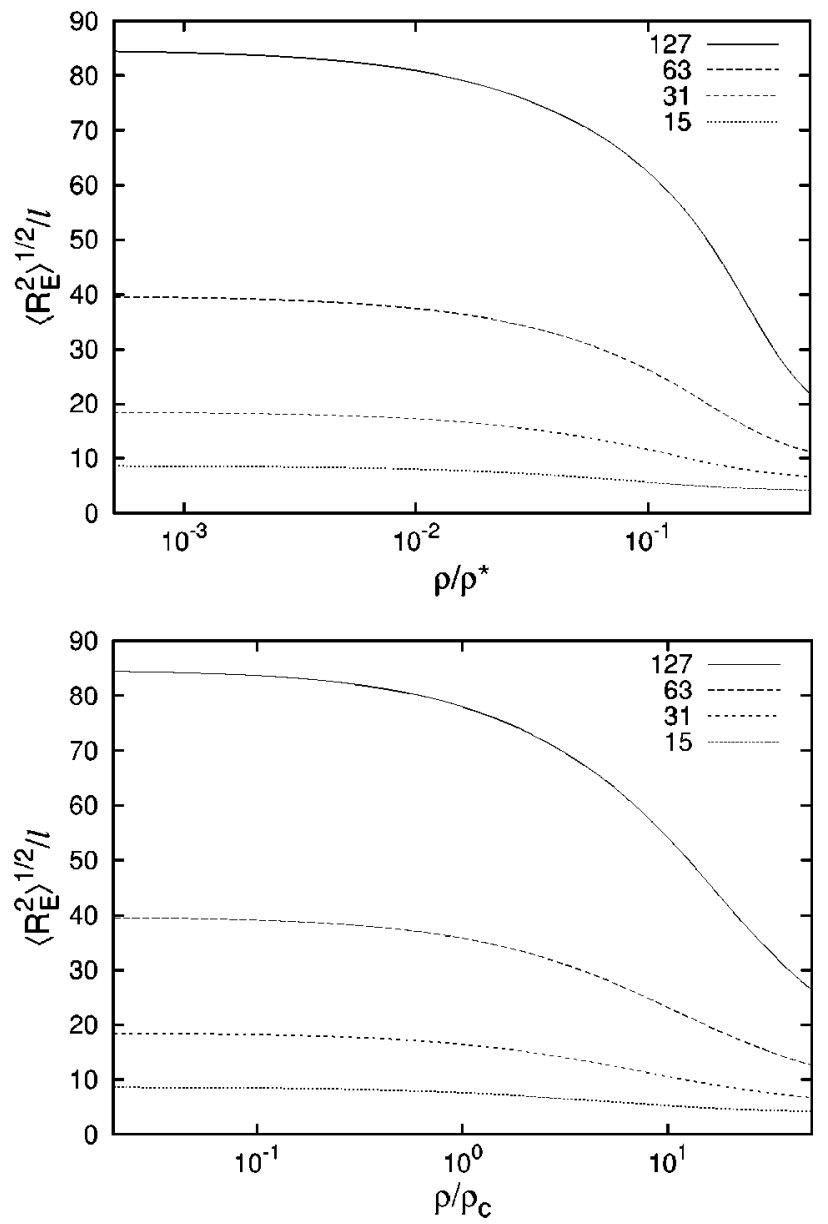

FIG. 4. Density dependence of the root mean square end-to-end distance for various flexible chains. The density is scaled by the overlap density $\rho^{*}$ (top) and the critical screening density $\rho_{c}$ (bottom), respectively. The Bjerrum length is $l_{\mathrm{B}}=0.833 l$.

in Fig. 3. With increasing Bjerrum length, we expect an increase of the chain extension. This is confirmed by the results presented in Fig. 5, where the mean square end-to-end distance is presented for various Bjerrum lengths ranging from $l_{\mathrm{B}}=0.1 l$ to $l_{\mathrm{B}}=2.0 l$. The figure exhibits the same qualitative behavior for all Bjerrum lengths, i.e., starting from a stretched conformation we find a decrease of the chain size with increasing density. At small densities, the size of a chain of given length increases rapidly with the Bjerrum length and almost reaches a fully stretched conformation with $\left\langle\mathbf{R}_{E}^{2}\right\rangle=N^{2} l^{2}$ for the highest Bjerrum length. The differences between the mean square end-to-end distances for the various Bjerrum lengths diminishes with increasing density. For very high densities the curves for the various Bjerrum lengths even assume the same value, which is given by the end-to-end distance of an uncharged chain. This behavior has to be expected, since for low densities the screening is so weak that an increase of the Bjerrum length leads to a stronger repulsion between the charges and therefore to a rapidly increasing chain size. For high densities, even the interaction between two adjacent charges is screened. Thus, the chain size becomes independent of the Bjerrum length and is given by the value of an uncharged chain. We like to mention that

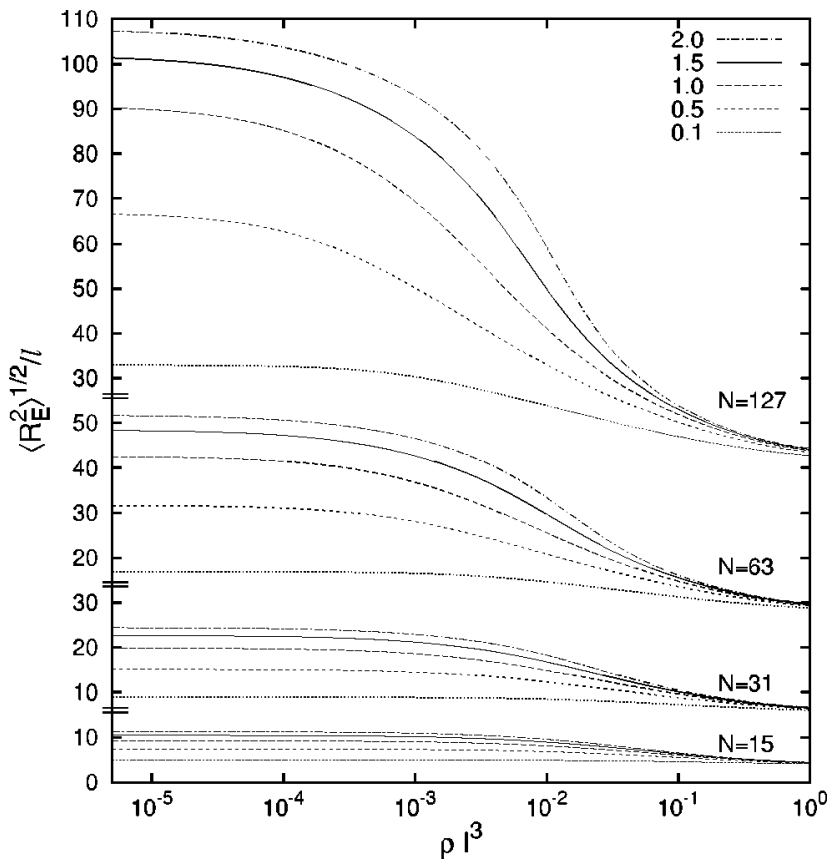

FIG. 5. Density dependence of the root mean square end-to-end distance for various Bjerrum lengths $\left(l_{\mathrm{B}} / l=0.1-2.0\right)$. The chain lengths of the flexible chains vary from $N=15$ to $N=127$.

the density where the chains start to contract is again well described by the screening density $\rho_{c}$.

The density dependence of the Lagrangian multiplier $\nu$ is shown in Fig. 6 for various Bjerrum lengths and the chain length $N=63$. The Lagrangian multiplier exhibits the same qualitative behavior as the mean square end-to-end distance, i.e., starting from a value which exceeds the value $\nu(0)$ $=\nu\left(l_{B}=0\right)$ of an uncharged chain, for all Bjerrum lengths the Lagrange multiplier drops to the value of the uncharged chain.

\section{B. Semiflexible chains}

The intrinsic stiffness, characterized by the parameter $t$ (4), significantly affects the conformation of a polyelectro-

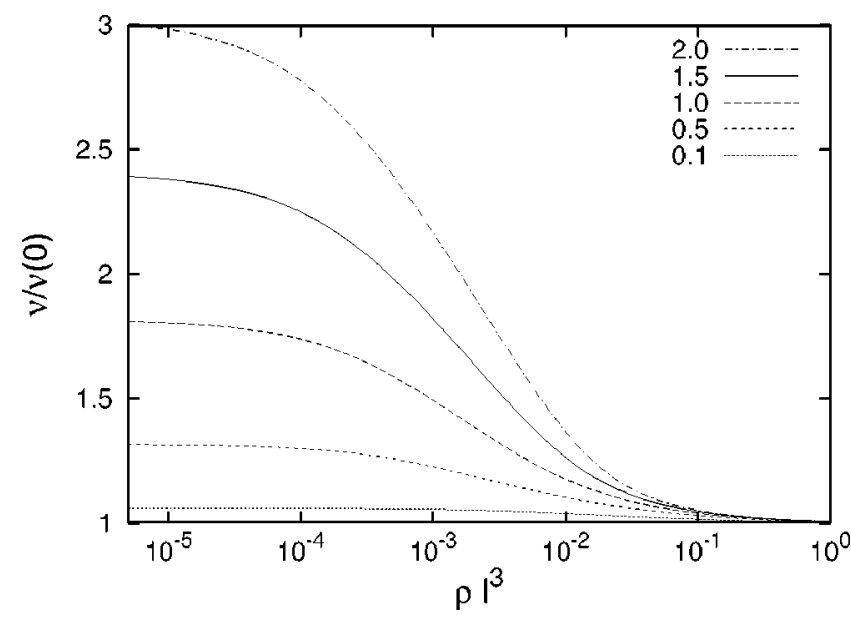

FIG. 6. Density dependence of the Lagrangian multiplier $\nu$ of flexible chains for various Bjerrum lengths and the chain length $N=63 . \nu(0)$ is the Lagrangian multiplier of an uncharged chain (5). 


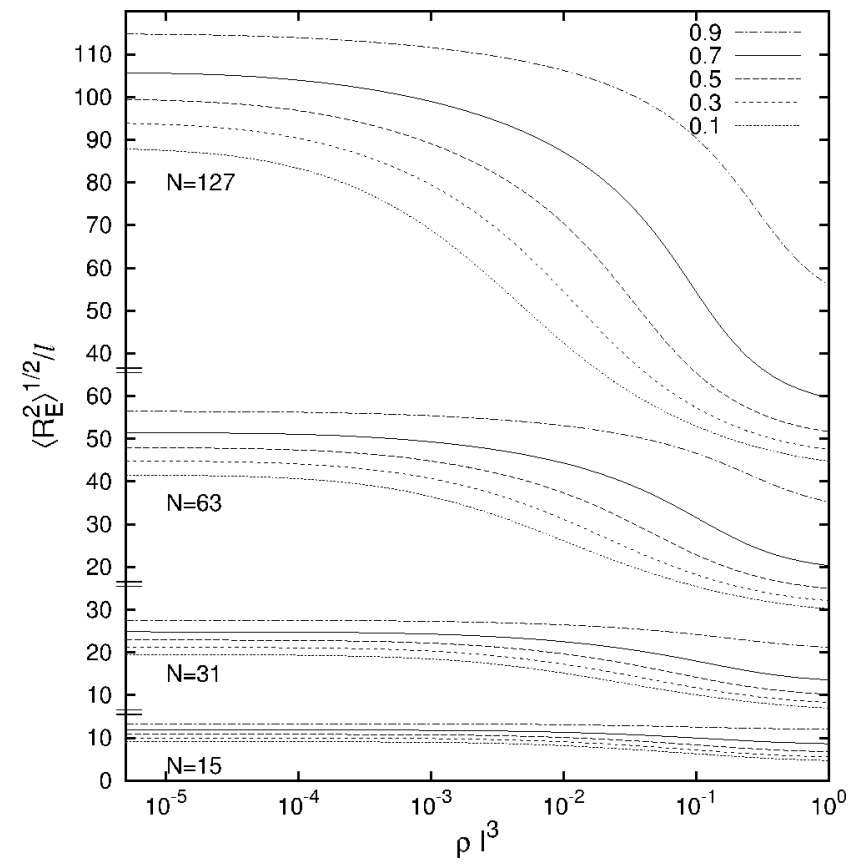

FIG. 7. Density dependence of the root mean square end-to-end distance of semiflexible chains with stiffnesses ranging from $t=0.1$ to $t=0.9$. The Bjerrum length is $l_{\mathrm{B}}=0.833 l$.

lyte chain in solution. Figure 7 displays the density dependence of the mean square end-to-end distance for various chain stiffnesses ranging from very flexible chains $(t=0.2)$ to very stiff chains $(t=0.9)$. The chain lengths are the same as in Fig. 3. Qualitatively, for all values of $t$ the same behavior is observed as for fully flexible chains. Considering the critical density, at which the chains start to contract, we find a shift to larger densities with increasing stiffness. This has to be expected, since in the limit $t=1$ of rodlike chains the mean square end-to-end distance has to be independent from the density, since no conformational changes are possible. Our recent computer simulations of semiflexible chains confirm this shift. The above results contradict the predictions presented in Ref. 54. The theory of Ref. 54 suggests a decrease of the critical density with increasing chain stiffness for semiflexible chains close to the flexible chain limit.

Moreover, Fig. 7 shows that the end-to-end distances of chains of larger stiffnesses always exceed those of chains with smaller stiffnesses. Even for the highest densities, larger variations are obtained. For flexible chains (cf. Sec. V A), we find that the chain sizes are similar to uncharged chains at high densities. Similarly, the mean square end-to-end distances of the semiflexible chains approach the corresponding values of the uncharged chains at high densities. This is displayed in Fig. 8, where the mean square end-to-end distances of the charged chains are scaled by the values of neutral chains. As the figure shows, the curves for the smaller chain stiffnesses are above those with higher stiffness, i.e., the Coulomb interaction stretches chains with smaller stiffness stronger than those with higher stiffness. This is explained by the fact that the chains with large values of $t$ require a larger force, i.e., a stronger Coulomb interaction, to change the

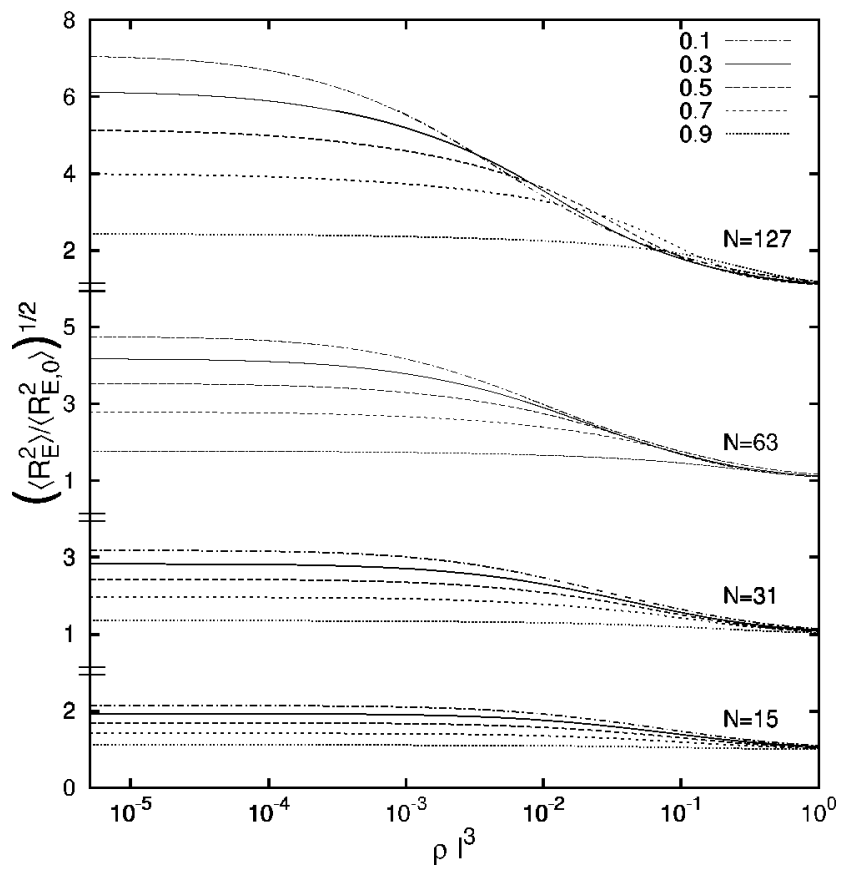

FIG. 8. Density dependence of the scaled root mean square end-to-end distance of semiflexible chains with stiffnesses ranging from $t=0.1$ to $t$ $=0.9$. The Bjerrum length is $l_{\mathrm{B}}=0.833 l .\left\langle\mathbf{R}_{E, 0}^{2}\right\rangle$ is the mean square end-toend distance of an uncharged semiflexible chain.

bond angles and therefore cannot be stretched as easily as the chains with smaller stiffness.

Reference 54 presents mean square end-to-end distances at high densities which assume the same value for all chain stiffnesses, i.e., the chains are compressed to a smaller size than given by the uncharged chain. This contradicts our results, where the medium-induced interaction balances the intramolecular Coulomb repulsion leading to conformations of an uncharged system at large densities. The discrepancies between the results suggests limitations in the applicability of the (simple) model presented in Ref. 54.

\section{Correlation function}

Insight into the structure of a polyelectrolyte solution can be gained by the pair correlation function $g(r)$. Figure 9 depicts the monomer pair correlation function for various densities and a chain length $N=63$. The stiffness ranges from a total flexible chain $(t=0)$ to a rodlike chain $(t=1)$, and the Bjerrum lengths are $l_{\mathrm{B}}=0.5 l$ [Fig. 9 (top)] and $l_{\mathrm{B}}$ $=1.5 l$ [Fig. 9 (bottom)], respectively. Semiflexible chains exhibit a pronounced liquidlike order with a peak, which shifts to smaller distances with increasing density, similar to the structure of rodlike systems. ${ }^{39-46}$ For the lowest density $\left(\eta=10^{-5}\right)$ the peak position and peak height are almost independent from the chain stiffness despite the fact that the chain size is very sensitive to the stiffness in this density range, as discussed in Sec. V B. This is explained by the fact that the structure of the liquid in this density range is dominated by electrostatic interactions and not packing effects, which would depend on the chain size. With increasing density the peak sharpens and its height changes. Moreover, the peak height decreases with increasing stiffness. Figure 9 

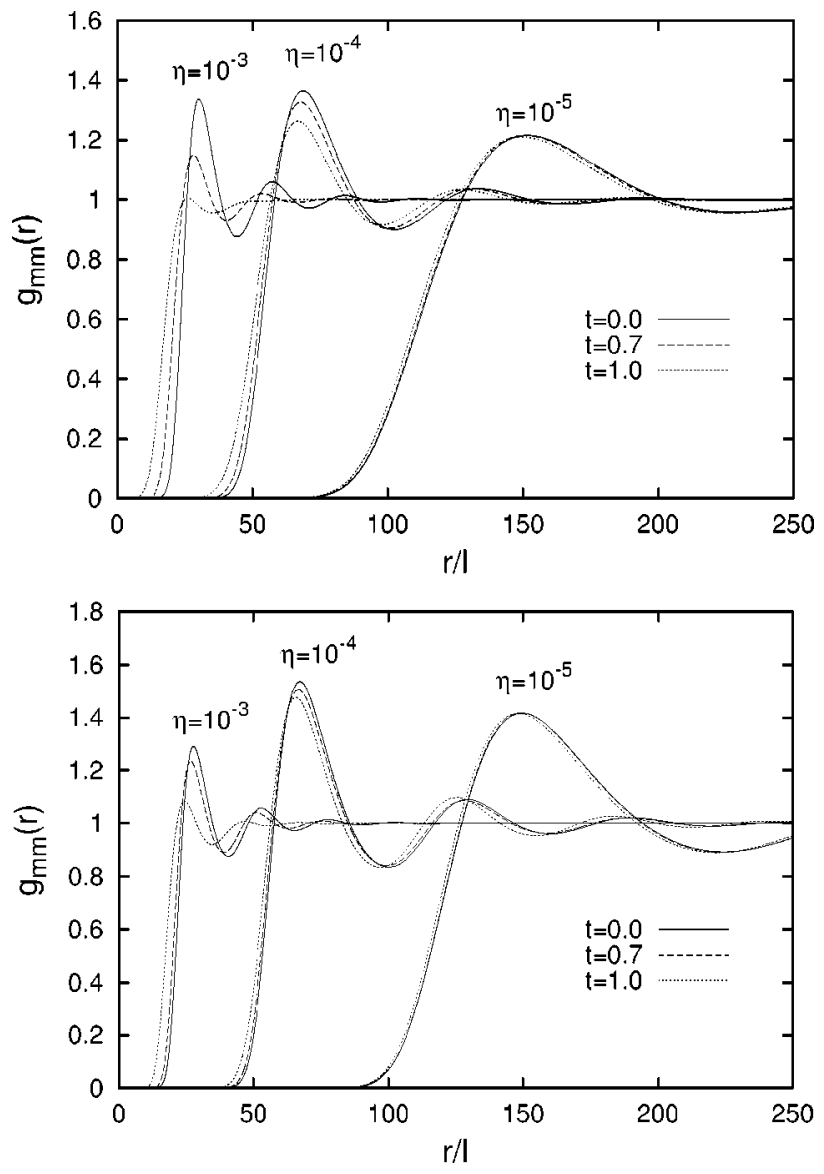

FIG. 9. Monomer-monomer pair correlation functions of semiflexible chains of various stiffnesses $t$ and densities $\eta$. The chain length is $N=63$ and the Bjerrum lengths are $l_{\mathrm{B}}=0.5 l$ (top) and $l_{\mathrm{B}}=1.5$ (bottom), respectively.

shows that this effect is more pronounced for higher densities. Therefore, an effect of the chain size should only be noticed for densities where packing effects have to be taken into account, i.e., for densities close and above the overlap density. For flexible chains, the peak maximum is always located at larger distances than for stiffer chains. At the same density, the coils of the flexible chains exhibit a smaller overlap and hence a stronger Coulomb interaction.

The comparison of Fig. 9 (top) and Fig. 9 (bottom) shows that the shift of the peaks and the increase of the peak height as a function of stiffness is less pronounced in the system with a larger Bjerrum length, especially for low and moderate densities. This results from the rodlike structure even of flexible chains at large Bjerrum lengths. Hence, the stiffness dependence of the correlation function disappears with an increasing Bjerrum length. However, this applies only as long as the screening length, which increases with increasing density, is not too small. The chains behave like uncharged chains for small screening lengths and hence exhibit again a dependence on the chain stiffness. Therefore, we still find large differences between the curves for the various stiffnesses at the highest density $\left(\eta=10^{-3}\right)$.

\section{CONCLUSIONS}

We have studied the conformational behavior and the structural properties of flexible and semiflexible polyelectro- lyte chains in solution using a self-consistent integral equation theory. We have extended the standard self-consistent theory by taking finite chain extensibility into account using Lagrangian multipliers.

The single chain results of our approach for the dependence of the mean square end-to-end distance on the Debye screening length or the density, respectively, are in excellent agreement with computer simulations. Considering the scaling behavior of the mean square end-to-end distance with respect to chain length, we find two different scaling regimes, corresponding to rodlike behavior and short-range excluded volume interactions, respectively. The range of chain lengths, where we observe nearly rodlike behavior, depends on the screening length and decreases with decreasing screening length. By increasing the density, the chains display a continuous transition from a stretched state to a collapsed state. We find that the collapse is governed by charge screening effects rather than packing effects, since the chains start to contract already at densities much smaller than the overlap density.

The calculation of the density dependence of the mean square end-to-end distance of flexible chains for various Bjerrum lengths shows that the end-to-end distance increases rapidly with the Bjerrum length for low densities. For high densities, however, the end-to-end distance is independent from the Bjerrum length due to the efficient screening and is similar to the value of an uncharged chain. Moreover, chain stiffness plays an important role for the size of the chains at all densities. The end-to-end distance is always larger for chains with large stiffness than for chains with low stiffness. However, at large densities, the end-to-end distances approach the values of the corresponding uncharged chains.

The presence of chain stiffness is also noticeable in the structural properties of a polyelectrolyte solution. Considering the pair correlation function, we find that semiflexible chains exhibit the same qualitative behavior as rodlike chains, but the typical peaks are shifted to larger distances and the height of the peaks increases with decreasing chain stiffness. This effect is more pronounced for lower densities and smaller Bjerrum lengths.

\section{ACKNOWLEDGMENTS}

The authors gratefully acknowledge the financial support by the Deutsche Forschungsgemeinschaft within the Schwerpunktsprogramm 1009.

\section{APPENDIX A: EDWARDS-SINGH APPROACH}

The Hamiltonians of the polyelectrolyte chain and the reference system are given by

$\beta H=\nu \sum_{i=2}^{N-1} \mathbf{R}_{i}^{2}+\nu_{0}\left(\mathbf{R}_{1}^{2}+\mathbf{R}_{N}^{2}\right)+\frac{\epsilon}{2} \sum_{i=1}^{N-1}\left(\mathbf{R}_{i}-\mathbf{R}_{i+1}\right)^{2}+\beta V(\mathbf{r})$,

$\beta H_{R}=\nu_{R} \sum_{i=2}^{N-1} \mathbf{R}_{i}^{2}+\nu_{0, R}\left(\mathbf{R}_{1}^{2}+\mathbf{R}_{N}^{2}\right)+\frac{\epsilon_{R}}{2} \sum_{i=1}^{N-1}\left(\mathbf{R}_{i}-\mathbf{R}_{i+1}\right)^{2}$, 
respectively. $V(\mathbf{r})$ is given by the potential (15). Inserting these terms into Eq. (10), the evaluation of the integrals yields the result

$$
0=\left(\nu-\nu_{R}\right) l^{2} A+\left(\nu_{0}-\nu_{0, R}\right) l^{2} B+\frac{1}{2}\left(\epsilon-\epsilon_{R}\right) l^{2} C+D,
$$

with

$$
\begin{aligned}
A= & \frac{2}{3\left(1-t_{R}\right)^{2}}\left(-N\left(\left(1+t_{R}\right)^{2}+2 t_{R} t_{R}^{N}\right)\right. \\
& \left.+2\left(1-t_{R}^{N}\right) \frac{\left(1-t_{R}^{N}\right)+2 t_{R}\left(1+t_{R}+t_{R}^{2}\right)}{1-t_{R}^{2}}\right), \\
B= & -\frac{4}{3}\left(\frac{t_{R}^{N}-1}{t_{R}-1}\right)^{2}, \\
C= & \frac{4}{3\left(1-t_{R}^{2}\right)}\left((N-1)\left(1-t_{R}^{2}\right) t_{R}^{N}+\left(t_{R}^{2 N}-t_{R}^{2}\right)\right), \\
D= & \frac{l^{2}}{18 \pi^{2}} \int_{0}^{\infty} d q q^{4} \widetilde{V}(q) \\
& \times \sum_{n=1}^{N} \exp \left(-\frac{q^{2}}{6} n l^{2}\left(\frac{1+t_{R}}{1-t_{R}}+\frac{2 t_{R}}{n} \frac{t_{R}^{n}-1}{\left(t_{R}-1\right)^{2}}\right)\right) \\
& \times\left\{n^{2}(N-n+1)\left(\frac{1+t_{R}}{1-t_{R}}\right)^{2}+8 t_{R}^{2} e^{2 N a} \frac{\sinh ^{2} a n}{\left(t_{R}-1\right)^{4}}\right. \\
& \times\left((N-n)-\frac{4 t_{R}}{1-t_{R}^{2}} \cosh a(N-n) \sinh a(N-n+2)\right) \\
& \left.-16 n t_{R}^{3 / 2} \frac{1+t_{R}}{\left(1-t_{R}\right)^{4}} e^{N a} \sinh a n \sinh a(N-n+1)\right\} .(A 7)
\end{aligned}
$$

$\widetilde{V}(q)$ is the Fourier transformed potential $V(\mathbf{r})$.

\section{APPENDIX B: STRETCHED SEMIFLEXIBLE CHAIN}

Stretching a semiflexible chain is achieved by the constraint

$$
\left\langle\mathbf{r}_{N}^{2}\right\rangle=\mathbf{a}^{2},
$$

for the chain end point $\left(\mathbf{r}_{N}\right)$. Applying the maximum entropy principle $^{67,70,80}$ and taking into account the constraints (1)(3), (B1), as well as the normalization condition of the distribution function yields the partition function

$$
\begin{aligned}
Z= & (\pi \sinh \vartheta)^{3 / 2}\left(\frac{2 \pi}{\epsilon}\right)^{3 N / 2} \\
& \times \Omega^{-3 / 2}\left(1+\eta \sum_{n=1}^{N} \sum_{m=1}^{N} A_{n m}^{-1}\right)^{-3 / 2},
\end{aligned}
$$

with the abbreviations ${ }^{62}$

$$
\begin{aligned}
\Omega= & \left(\frac{2 \widetilde{\nu}_{0}+\epsilon}{\epsilon}\right)^{2} \sinh (N-1) \vartheta-2 \frac{2 \widetilde{\nu}_{0}+\epsilon}{\epsilon} \sinh (N-2) \vartheta \\
& +\sinh (N-3) \vartheta
\end{aligned}
$$

$$
\begin{aligned}
A_{n m}^{-1}= & \frac{2}{\epsilon \Omega \sinh \vartheta}\left[\frac{2 \widetilde{\nu}_{0}+\epsilon}{\epsilon} \sinh (n-1) \vartheta-\sinh (n-2) \vartheta\right] \\
& \times\left[\frac{2 \widetilde{\nu}_{0}+\epsilon}{\epsilon} \sinh (N-m) \vartheta-\sinh (N-m-1) \vartheta\right],
\end{aligned}
$$

$$
\cosh \vartheta=\frac{\widetilde{v}+\epsilon}{\epsilon} .
$$

[In Eq. (B4) $n<m$.] The Lagrangian multipliers follow from the conditions $\partial \ln Z / \partial \lambda=-\phi$, where $\lambda \in \widetilde{\nu}, \widetilde{\nu}_{0}, \eta$ and $\phi$ is the corresponding expectation value $\left(\phi \in(N-2) l^{2}, l^{2}, \mathbf{a}^{2}\right)$. Explicitly, we find for $\eta$,

$$
\eta=\frac{3}{2}\left(\frac{1}{a^{2}}-\frac{1}{\left\langle\mathbf{R}_{E}^{2}\right\rangle_{0}}\right)
$$

where $\left\langle\mathbf{R}_{E}^{2}\right\rangle_{0}$ is the mean-square end-to-end distance of a nonstretched chain given by

$$
\begin{aligned}
\left\langle\mathbf{R}_{E}^{2}\right\rangle_{0}= & \frac{3(N+2)}{2 \widetilde{\nu}}-\frac{3 \widetilde{\nu}_{0}}{\widetilde{\nu}^{2}}+\frac{6}{\epsilon}\left(\frac{\widetilde{\nu}_{0}}{\widetilde{\nu}}-1\right)^{2} \\
& \times\left[\frac{2 \widetilde{\nu}_{0}+\epsilon}{\epsilon} \sinh (N-1) \vartheta-\sinh (N-2) \vartheta\right. \\
& +\sinh \vartheta] / \Omega .
\end{aligned}
$$

The Lagrange multipliers $\widetilde{\nu}$ and $\widetilde{\nu}_{0}$ have to be calculated numerically from the following equations:

$$
\sum_{n=2}^{N-1}\left[A_{n, n}-\frac{\eta}{1+\frac{2}{3} \eta\left\langle\mathbf{R}_{E}^{2}\right\rangle_{0}}\left(\sum_{i=1}^{N} A_{i, n}\right)^{2}\right]=\frac{2}{3}(N-2) l^{2},
$$

$$
A_{1,1}-\frac{\eta}{1+\frac{2}{3} \eta\left\langle\mathbf{R}_{E}^{2}\right\rangle_{0}}\left(\sum_{i=1}^{N} A_{i, 1}\right)^{2}=\frac{2}{3} l^{2} .
$$

Detailed results for a totally flexible chain $(t=0)$ are presented in Ref. 67.

\footnotetext{
${ }^{1}$ M. Mandel, Polyelectrolytes (Reidel, Dordrecht, 1988).

${ }^{2}$ M. Hara, Polyelectrolytes: Science and Technology (Dekker, New York, 1993).

${ }^{3}$ F. Buchholz, Trends Polym. Sci. 99, 277 (1982).

${ }^{4}$ A. Bhattacharya, Prog. Polym. Sci. 25, 371 (2000).

${ }^{5}$ S. Förster and M. Schmidt, Adv. Polym. Sci. 120, 51 (1995).

${ }^{6}$ C. Tanford, Physical Chemistry of Macromolecules (Wiley, New York, 1961).

${ }^{7}$ H. F. Mark, Encyclopedia of Polymer Science and Engineering (Wiley, New York, 1990).

${ }^{8}$ J.-L. Barrat and J.-F. Joanny, Adv. Chem. Phys. XCIV, 1 (1996).

${ }^{9}$ K. Schmitz, Macroion Characterization: From Dilute Solutions to Complex Fluids (American Chemical Society, Washington, 1994).

${ }^{10}$ F. B.-W. J. Hayter, G. Janninck, and P. G. de Gennes, J. Phys. (Paris) 41, 451 (1980).

${ }^{11}$ L. Wang and V. Bloomfield, Macromolecules 23, 315 (1990).

${ }^{12}$ T. Witten and P. Pincus, Europhys. Lett. 3, 315 (1987).

${ }^{13}$ P. G. de Gennes, Scaling Concepts in Polymer Physics (Cornell University Press, Ithaca, NY, 1979).

${ }^{14}$ J. des Cloiseaux and G. Jannink, Les Polymeres en Solution, Leur Modelisation et Leur Structure (Editions de Physique, Paris, 1985).
} 
${ }^{15}$ M. Doi and S. F. Edwards, The Theory of Polymer Dynamics (Oxford University Press, Oxford, 1986).

${ }^{16}$ M. Deserno and C. Holm, J. Chem. Phys. 109, 7694 (1998).

${ }^{17}$ C. Holm, K. Kremer, and T. A. Vilgis, Phys. Bl. 54, 1013 (1998).

${ }^{18}$ U. Micka, C. Holm, and K. Kremer, Langmuir 15, 4033 (1999).

${ }^{19}$ M. Stevens and K. Kremer, Phys. Rev. Lett. 71, 2228 (1993).

${ }^{20}$ M. Stevens and K. Kremer, J. Chem. Phys. 103, 1669 (1995).

${ }^{21}$ R. G. Winkler, M. Gold, and P. Reineker, Phys. Rev. Lett. 80, 3731 (1998).

${ }^{22}$ R. Chang and A. Yethiraj, J. Chem. Phys. 116, 5284 (2002).

${ }^{23}$ Y. Hayashi, M. Ullner, and P. Linse, J. Chem. Phys. 116, 6836 (2002).

${ }^{24}$ S. Liu and M. Muthukumar, J. Chem. Phys. 116, 9975 (2002).

${ }^{25}$ R. G. Winkler, M. O. Steinhauser, and P. Reineker, Phys. Rev. E 66, 021802 (2002).

${ }^{26}$ J. Bodycomb and M. Hara, Macromolecules 27, 7369 (1994).

${ }^{27}$ M. Nierlich, C. E. Williams, F. Boue et al., J. Phys. (Paris) 40, 701 (1979).

${ }^{28}$ M. Nierlich, F. Boue, A. Lapp, and R. Oberth, Colloid Polym. Sci. 263, 955 (1985).

${ }^{29}$ G. Jannink, Makromol. Chem., Macromol. Symp. 1, 67 (1986).

${ }^{30}$ M. Sedlak and E. Amis, J. Chem. Phys. 96, 817 (1992).

${ }^{31}$ S. Förster, M. Schmidt, and M. Antonietti, J. Phys. Chem. 96, 4008 (1992).

${ }^{32}$ L. Wang and V. Bloomfield, Macromolecules 24, 5791 (1991).

${ }^{33}$ H. Miura and A. D. English, Macromolecules 21, 1544 (1988)

${ }^{34}$ S. F. Schulz, E. E. Maier, R. Krause, M. Hagenbüchle, M. Deggelmann, and R. Weber, J. Chem. Phys. 92, 7087 (1990).

${ }^{35}$ C. Graf, M. Deggelmann, M. Hagenbüchle, H. Kramer, R. Krause, C. Martin, and R. Weber, J. Chem. Phys. 95, 6284 (1991).

${ }^{36}$ K. S. Schweizer and J. G. Curro, J. Chem. Phys. 91, 5059 (1989).

${ }^{37}$ K. S. Schweizer and A. Yethiraj, J. Chem. Phys. 98, 9053 (1993).

${ }^{38}$ J. P. Hansen and I. R. McDonald, Theory of Simple Liquids (Academic, London, 1986).

${ }^{39}$ M. Dymitrwska and L. Belloni, J. Chem. Phys. 111, 6633 (1999).

${ }^{40}$ L. Harnau and P. Reineker, J. Chem. Phys. 112, 437 (2000).

${ }^{41}$ T. Hofmann, R. G. Winkler, and P. Reineker, J. Chem. Phys. 114, 10181 (2001).

${ }^{42}$ A. Yethiraj and C. Y. Shew, Phys. Rev. Lett. 77, 3937 (1996).

${ }^{43}$ C. Y. Shew and A. Yethiraj, J. Chem. Phys. 110, 5437 (1999).

${ }^{44}$ C. Y. Shew and A. Yethiraj, J. Chem. Phys. 106, 5706 (1997).

${ }^{45}$ C. Y. Shew and A. Yethiraj, J. Chem. Phys. 109, 5162 (1998).

${ }^{46}$ C. Y. Shew and A. Yethiraj, J. Chem. Phys. 110, 11599 (1999).

${ }^{47}$ J. R. J. P. Donley and A. Liu, Macromolecules 30, 1188 (1997).

${ }^{48}$ A. Yethiraj and K. S. Schweizer, J. Chem. Phys. 97, 1455 (1992).

${ }^{49}$ K. S. Schweizer, K. G. Honnell, and J. G. Curro, J. Chem. Phys. 96, 3211 (1992).
${ }^{50}$ J. Melenkevitz and J. G. Curro, J. Chem. Phys. 99, 5571 (1993).

${ }^{51}$ C. J. Grayce, A. Yethiraj, and K. S. Schweizer, J. Chem. Phys. 100, 6857 (1994).

${ }^{52}$ A. Yethiraj, Phys. Rev. Lett. 78, 3789 (1997).

${ }^{53}$ A. Yethiraj, J. Chem. Phys. 108, 1184 (1998).

${ }^{54}$ C. Y. Shew and A. Yethiraj, J. Chem. Phys. 113, 8841 (2000).

${ }^{55}$ J. G. Curro, K. S. Schweizer, G. S. Grest, and K. Kremer, J. Chem. Phys. 91, 1357 (1989).

${ }^{56}$ K. S. Schweizer and J. G. Curro, Adv. Chem. Phys. XCVIII, 1 (1997).

${ }^{57}$ D. Chandler, Y. Singh, and D. M. Richardson, J. Chem. Phys. 81, 1975 (1984).

${ }^{58}$ D. Laria, D. Wu, and D. Chandler, J. Chem. Phys. 95, 4444 (1991).

${ }^{59}$ J. Melenkevitz, K. S. Schweizer, and J. G. Curro, Macromolecules 26, 6190 (1993).

${ }^{60}$ Y. Singh, J. Phys. A 20, 3949 (1987)

${ }^{61}$ K. Ghosh, G. A. Carri, and M. Muthukumar, J. Chem. Phys. 115, 4367 (2001).

${ }^{62}$ R. G. Winkler, P. Reineker, and L. Harnau, J. Chem. Phys. 101, 8119 (1994).

${ }^{63}$ M. G. Bawendi and K. F. Freed, J. Chem. Phys. 83, 2491 (1985).

${ }^{64}$ S. M. Battacharjee and M. Muthukumar, J. Chem. Phys. 86, 411 (1987).

${ }^{65}$ J. B. Lagowski, J. Noolandi, and B. Nickel, J. Chem. Phys. 95, 1266 (1991).

${ }^{66}$ B.-Y. Ha and D. Thirumalai, J. Chem. Phys. 103, 9408 (1995).

${ }^{67}$ R. G. Winkler and P. Reineker, Macromolecules 25, 6891 (1992).

${ }^{68}$ R. G. Winkler and P. Reineker, J. Chem. Phys. 106, 2841 (1997).

${ }^{69}$ T. Hofmann, R. G. Winkler, and P. Reineker, Phys. Rev. E 61, 2840 (2000).

${ }^{70}$ R. G. Winkler, J. Chem. Phys. 118, 2919 (2003).

${ }^{71}$ B. Y. Ha and D. Thirumalai, J. Chem. Phys. 110, 7533 (1999).

${ }^{72}$ S. F. Edwards and P. Singh, J. Chem. Soc., Faraday Trans. 2 75, 1001 (1979).

${ }^{73}$ R. R. Netz and H. Orland, Eur. Phys. J. B 8, 81 (1999).

${ }^{74}$ K. S. Schweizer and J. G. Curro, Macromolecules 21, 3070 (1988).

${ }^{75}$ A. Yethiraj and C. K. Hall, J. Chem. Phys. 93, 4453 (1990).

${ }^{76}$ A. Yethiraj and C. K. Hall, J. Chem. Phys. 96, 797 (1992).

${ }^{77}$ A. L. Nichols, D. Chandler, Y. Singh, and D. M. Richardson, J. Chem. Phys. 81, 5109 (1984)

${ }^{78}$ M. Muthukumar, J. Chem. Phys. 86, 7230 (1987).

${ }^{79}$ T. T. Nguyen and B. I. Shklovskii, Phys. Rev. E 66, 021802 (2002).

${ }^{80} \mathrm{H}$. Haken, Synergetics (Springer-Verlag, Berlin, 1983).

${ }^{81}$ M. Stevens and K. Kremer, Macromolecules 26, 4717 (1993). 